\title{
Arqueología de las zonas de estudio de un proyecto de Teledetección en la República de Honduras
}

\author{
Vito Veliz , Arnulfo Ramírez, César Rodríguez, \\ Yenny Castellanos, Rafael Corrales \\ y Juan Gregorio Rejas Ayuga
}

\section{Resumen}

Con este proyecto se pretende aplicar tecnologías digitales recientes para identificar, ubicar y registrar restos de patrimonio cultural, especialmente los prehispánicos. Una vez identificados esos restos y estudiado el entorno, se espera desarrollar programas contra riesgos naturales y humanos. Con estas prácticas se espera predecir zonas de peligro y proponer usos diferentes de la tierra para mejorar la protección de los restos arqueológicos. Ya que el proyecto se irá desarrollando por etapas, se espera con el tiempo tener suficiente información para relacionar estructuras dentro de los sitios arqueológicos y también poder llegar a relacionar sitios arqueológicos entre zonas y tratar de encontrar así, si es que existen, las relaciones entre estructuras y entre sitios con los puntos cardinales y con los astros.

Palabras claves: arqueologia, teledeteccion, patrimonio cultural, Honduras

\section{Abstract}

This project aims to apply recent digital technologies to identify, locate and register remains of cultural heritage, especially the pre-Hispanic. Once identified the remains and studied the environment, it is expected to develop programs against natural and human hazards. These practices are expected to predict danger areas and propose different land uses to improve the protection of the archaeological remains. Since the project will develop in stages, it is expected eventually to have sufficient information to relate structures within archaeological sites and also to reach related archaeological sites between areas and try to find as well, if there exist, relations between structures and between sites with the cardinal points and the stars.

Keywords: archaeology, remote sensing, cultural heritage, Honduras 
Vito Veliz ${ }^{1}$, Arnulfo Ramírez ${ }^{1}$, César Rodríguez ${ }^{1}$, Yenny Castellanos ${ }^{2}$, Rafael Corrales ${ }^{2}$ y Juan Gregorio Rejas Ayuga ${ }^{3}$. 'Departamento de Arqueoastronomía y Astronomía Cultural. ${ }^{2}$ Departamento de Ciencias y Tecnologías de la Información Geográfica, Facultad de Ciencias Espaciales, Universidad Nacional Autónoma de Honduras. ${ }^{3}$ Depto. de Ingeniería y Morfología del Terreno, Universidad Politécnica de Madrid, UPM. 


\section{Introducción}

Después de haber escuchado sobre la identificación de distintos objetos 0 fenómenos en la superficie terrestre por medio de imágenes satelitales, la en aquel entonces Sección de Arqueoastronomía del Observatorio Astronómico Centroamericano de Suyapa de la Universidad Nacional Autónoma de Honduras (OACS/ UNAH) propuso verbalmente a la Universidad Politécnica de Madrid la idea de desarrollar un proyecto para identificar sitios arqueológicos en Honduras, restos que son parte de nuestro patrimonio cultural.

La idea fue aceptada y el Profesor Juan Gregorio Rejas Ayuga presentó el proyecto ante las autoridades españolas, teniendo también el aval de las autoridades de la UNAH, incluyendo a la Vicerrectoría de Relaciones Internacionales y a la Dirección del OACS. En vista de que el patrimonio cultural hondureño está bajo el cuidado y resguardo del Instituto Hondureño de Antropología e Historia (IHAH), también a esta institución se le invitó a participar. Esta participación sería en doble sentido: por un lado, contando con la participación de profesionales y/o técnicos en el proyecto; por otro, proporcionando al OACS la información pertinente sobre sitios arqueológicos ya conocidos. El proyecto en general podría así acrecentar la lista de sitios arqueológicos que el IHAH ha venido conformando.

La pretensión de esa lista de sitios arqueológicos es finalmente lograr un Atlas Arqueológico de Honduras, que pueda servir a propios y extraños para la investigación. Ésta es una tarea que el IHAH ha venido desarrollando a través de varios años y nuestro proyecto busca avanzar y ampliar la información. Es la intención trabajar con las tarjetas que ya ha elaborado el $\mathrm{HAH}$ y, de ser posible, mejorarlas para avanzar en ese registro.

Se comenzó a elaborar la lista de sitios arqueológicos, partiendo de una división temporal (Véliz, 1983) de las investigaciones arqueológicas en Honduras. Para poner el trabajo de Véliz al día, se fijaron seis períodos para lograr esa lista de yacimientos arqueológicos en Honduras. Se comenzó así a elaborar la lista de los sitios arqueológicos que cada investigador registró en su momento. Se continúa trabajando y parte de ese esfuerzo son los sitios arqueológicos que, junto con los miembros de CTIG (Departamento de Ciencias y Tecnologías de la Información Geográfica), (Rafael Corrales y Jenny Castellanos), se han logrado insertar en una tabla con información mínima. 
Los Objetivos de esta investigación se enmarcan en la importancia del uso de Sistemas de Información Geográfica en Arqueología y su futura aplicación en Honduras.

La metodología empleada se basa en una amplia revisión bibliográfica, análisis de documentos y registro de sitios arqueológicos encontrados procurando su ubicación geográfica, el uso de SIG y prospección de campo para validación y toma de puntos de interés para la investigación.

Se presentan en esta investigación definiciones y conceptos relacionados con la investigación arqueológica en Honduras y su estado legal.

Se espera que los resultados de esta investigación nos permitan comprobar la noción acerca del gran potencial de Honduras para investigaciones arqueológicas.

La selección de áreas de estudio en Honduras para la aplicación de Nuevas Tecnologías de la Información Geográfica, nos da una idea de algunas regiones seleccionadas de acuerdo a su potencial arqueológico e influencia cultural, siendo hasta ahora desconocidos muchos de los sitios a investigar.

Se destaca en este documento: El futuro de las investigaciones arqueológicas mediante Nuevas Tecnologías de la Información Geográfica en Honduras, lo cual nos permitirá estudiar amplias e importantes zonas del país por medio de tecnología satelital, contribuyendo a la realización de estos estudios que normalmente representan un alto costo económico en el campo.

\section{Objetivos}

\section{Principal:}

Desarrollar y aplicar metodologías no intrusivas, empleando tecnologías de detección y registro digitales, integradas en sistemas de información, para estudio y para mejorar la protección del patrimonio cultural y de los hábitats humanos de Honduras ante riesgos y desastres naturales y humanos.

\section{Específicos:}

- Identificación y localización de restos o sitios arqueológicos.

- Registro digital de los sitios arqueológicos. 
- Identificación de lugares de valor patrimonial amenazados por causas naturales o humanas.

\section{Metodología}

Se ha comenzado con la revisión de literatura para ubicar sitios arqueológicos ya conocidos y se ha continuado con el registro digital de esos sitios arqueológicos. Se pondrá especial interés en el registro de nuevos sitios arqueológicos. Los modelos de predicción, en cuanto a la existencia de sitios arqueológicos, que se propongan en base al estudio de las imágenes satelitales, se validarán en el campo. Se realizará la prospección arqueológica por medio de técnicas no destructivas, mediante sensores (espaciales, aeroportados y de rango corto) y GPR (Ground Penetration Radar), para la detección y el registro de restos culturales debajo de la superficie terrestre. Posteriormente se identificará las áreas vulnerables, utilizando datos de los Sistemas de Información Geográfica y de muestreos en el campo. Se propondrá así usos alternativos de la tierra para mejorar la protección del patrimonio ante situaciones de riesgo o desastre, tanto natural como humano.

La búsqueda de relación entre estructuras, sitios, puntos cardinales y astros, se realizará ubicando todos estos datos en mapas y trazando líneas para encontrar estas relaciones, si es que existen. Por fin, los resultados de estos esfuerzos se darán a conocer al público por medio de charlas y de publicaciones escritas de los resultados.

\section{DEFINICIONES Y CONCEPTOS RELACIONADOS CON LA INVESTIGACION}

A continuación se presentan una serie de definiciones de Patrimonio Cultural y Patrimonio Arqueológico, tanto de la UNESCO como de la legislación hondureña. Es importante poder conocer estos conceptos que apoyan nuestra investigación, sentando las bases teóricas en esta línea de investigación muy desconocida y de interés nacional, por lo que se incluyen en este artículo para apoyo y entendimiento del trabajo realizado.

\section{- Patrimonio Cultural}

En general, patrimonio es lo que uno posee o ha heredado.

Según la UNESCO, el patrimonio cultural lo componen conjuntos y lugares, obras arquitectónicas, de escultura 0 de pintura monumentales, elementos 0 estructuras de carácter arqueológico, inscripciones, cavernas y grupos de elementos, 
que tengan un valor universal excepcional desde el punto de vista de lo histórico, estético, etnológico, artístico y antropológico.

Expresándolo de otra manera, la UNESCO dice que el patrimonio cultural es el conjunto de objetos 0 artefactos, significados, convenciones que constituyen la expresión de una cultura especifica y que destacan sea porque no hay muchos, por la artesanía con la que fueron elaborados o porque poseen características únicas de tal cultura.

La Constitución de la República de Honduras, Capítulo VIII, Artículo 172, dice: "Toda riqueza antropológica, arqueológica, histórica y artística de Honduras forma parte del patrimonio cultural de la Nación.

La ley establecerá las normas que servirán de base para su conservación, restauración, mantenimiento y restitución, en su caso.

Es deber de todos los hondureños velar por su conservación e impedir su sustracción.

Los sitios de belleza natural, monumentos y zonas reservadas, estarán bajo la protección del Estado".

El Artículo 173 dice: "El Estado preservará y estimulará las culturas nativas, así como las genuinas expresiones del folklore nacional, el arte popular y las artesanías".

En sus considerandos, la Ley para la Protección del Patrimonio Cultural de la Nación (Decreto No. 220-97) dice que conforman el Patrimonio Cultural de la Nación los bienes culturales que poseen especialmente valor por su importancia histórica y antropológica.

También dice que los bienes culturales constituyen uno de los fundamentos de la cultura de los pueblos y que adquieren su verdadero valor cuando se conocen con precisión su origen, historia y contexto y se divulgan para el conocimiento de la población.

Menciona además que la Conferencia General de la UNESCO aprobó, en 1964, una recomendación con este objeto; y que la misma Conferencia General en su $16^{a}$ reunión, celebrada en París, en noviembre de 1970, aprobó la Convención Sobre Medidas que deben adoptarse para la Protección del Patrimonio Cultural de las Naciones. 
En su Capítulo I, Artículo 1, esta Ley para la Protección del Patrimonio Cultural dice que "tiene por objeto la defensa, conservación, reivindicación, rescate, restauración, protección, investigación, divulgación, acrecentamiento y transmisión a las generaciones futuras de los bienes que constituyen el Patrimonio Cultural de la Nación en todo el territorio nacional y en las aguas jurisdiccionales".

\section{- Patrimonio Arqueológico}

De acuerdo con el Capítulo II de la Ley de Patrimonio, Artículo 2, "se considera que forma parte del Patrimonio Cultural:

- Los Monumentos: Aquellos bienes inmuebles de la época precolombina, colonial y republicana que por su arquitectura o ingeniería sean de interés antropológico histórico.

- Bienes Muebles: Grabados, pinturas, esculturas, mobiliario, joyería, moneda, armas, vestuario, máquinas, herramientas u otros objetos de interés antropológico e histórico.

- Los Conjuntos: Agrupación de bienes inmuebles y su entorno natural que forman un patrón de asentamiento, continuo o disperso, que puede ser claramente delimitado, condicionado por una estructura física representativa de la evolución de una comunidad humana, por ser testimonio de su cultura.

- Sitio Arqueológico: Aquella área o lugar abandonado que presenta evidencias de actividad humana en forma de artefactos, rasgos y/o alteraciones producto de la misma, sean éstas de época precolombina, colonial o republicana de interés antropológico e histórico e incluyendo las evidencias que se encuentran en aguas jurisdiccionales en la superficie y en el subsuelo.

- Zona Arqueológica: Es un lugar donde existe un conjunto o grupo de sitios arqueológicos.

- Las Manifestaciones Culturales de los pueblos indígenas vivos, sus lenguas, sus tradiciones históricas, sus conocimientos y técnicas, sus formas de organización, sus sistemas de valores, sus prácticas religiosas y los lugares asociados a ellas; $y$,

- Las Manifestaciones Culturales de origen vernáculo vivas que sean de interés antropológico e histórico, organizaciones y celebraciones 
religiosas, música y danza, los prototipos de la producción artesanal y del arte culinario, la tradición oral.

Para nuestro interés, en su Artículo 7, el Reglamento para la Investigación Arqueológica en Honduras agrega una categoría más a las diez anteriores: Parque Arqueológico. Éste se define como "Monumento, conjunto o sitio arqueológico previamente declarado; con fines de conservación, investigación, educación y visitación".

Volviendo a la Ley de Patrimonio, en ese mismo Capítulo II, Artículo 3, se dice que "los bienes culturales protegidos que integran el Patrimonio Cultural Nacional se clasifican de la manera siguiente:

- Bienes Nacionales Culturales de Uso Público, entendiéndose como tales:

- La totalidad del Patrimonio precolombino;

- El patrimonio cultural sumergido; $y$,

- Los fondos documentales y bibliográficos de uso público;

- Bienes culturales propiedad de instituciones eclesiásticas.

- Bienes culturales propiedad de particulares que formen parte del patrimonio personal o familiar o hayan sido obtenidos lícitamente en su momento; $y$,

- Bienes de cultura popular, que son propiedad de las comunidades que los producen".

En ese mismo Capítulo II, Artículo 4, también dice que "para la adecuada defensa del Patrimonio Cultural, el Estado de Honduras declara el dominio o propiedad permanente, inalienable, imprescriptible y no comerciable sobre los bienes a los que se refiere el numeral 1) del Artículo 3 de esta Ley.

\section{Inventario}

Para el registro e inventario de los bienes culturales, el Decreto 220-97 estipula en su Capítulo IV, Artículo 5, que "el Instituto Hondureño de Antropología e Historia elaborará y mantendrá al día un inventario nacional de los bienes que constituyen el Patrimonio Cultural y tendrá la obligación de resguardarlos....". 
En el Artículo 13 dice que el "Patrimonio Cultural deberá estar debidamente registrado en el Instituto Hondureño de Antropología e Historia, caso contrario, se tendrá como posesión ilícita y deberá ser recuperado y administrado....".

El Capítulo V se refiere a los particulares propietarios de bienes culturales en los siguientes términos: "Toda persona natural o jurídica que esté en posesión legítima de bienes nacionales culturales...se considera depositaria temporal y responsable de su conservación y custodia.... (Artículo 14).

Se menciona también que el Instituto Hondureño de Antropología e Historia en coordinación con el Instituto Geográfico Nacional emitirá las disposiciones para conservar el acervo toponímico, en las lenguas indígenas originales y rescatar aquellos nombres tradicionales indígenas y de origen colonial que hayan caído en desuso o hayan sido suplantados por intervención de cualquier dependencia del Estado o de particulares (Artículo 55).

\section{Valorización del Patrimonio Cultural}

Para la valorización del patrimonio cultural se toman en cuenta cuatro valores fundamentales: Comunidad, familia, país y mundo entero. A la suma de estos valores se le conoce como significado cultural. En la Ley de Patrimonio Cultural se expresan estos valores como valor antropológico y valor histórico. El valor antropológico se deriva de la información que todo tipo de cultura material e inmaterial (objeto, sitio o manifestación cultural) brinda a la arqueología o a la etnología. El valor histórico se desprende de la importancia que tiene un objeto, sitio o manifestación para dar información sobre el cambio de una sociedad a través del tiempo.

\section{Clasificación de Sitios Arqueológicos}

Willey fue el pionero en cuanto a la clasificación de sitios arqueológicos en Honduras (Willey y Leventhal 1979: 81-83). Para esa clasificación se tomó en cuenta solamente el tamaño de los montículos y la complejidad de los grupos de montículos. Pero ni él ni los siguientes investigadores han tomado en cuenta los lineamientos de valorización de la UNESCO ni de otros organismos, si es que aplican.

Por lo que se encuentra en la documentación revisada, hasta el momento, en ningún documento se define el valor de un bien cultural. Los arqueólogos han producido clasificaciones, pero basándose en lo que aflora en la superficie. Así, en el Proyecto La Entrada (Nakamura, Aoyama y Uratsuji 1991, Tomo I: 12-13), por 
ejemplo, lo que toman en cuenta para la clasificación de los sitios arqueológicos son los siguientes criterios:

- Tamaño

- Ordenamiento Arquitectónico

- Complejidad

- Extensión

- Presunta Función

En base a esos criterios, las categorías de sitios arqueológicos que se definieron en La Entrada, son las siguientes (Nakamura, Aoyama y Uratsuji 1991, Tomo I: 13-14):

\section{Categoría 1}

Artefactos dispersos en la superficie, sin estructuras presentes.

\section{Categoría 2}

Una estructura aislada o agrupamientos de estructuras de menos de $2 \mathrm{mts}$. de altura. Algunos agrupamientos tienen patios, los que se supone son unidades habitacionales de campesinos.

\section{Categoría 3}

Se definen por las siguientes características:

- Estructura más alta mide aproximadamente de 2 a $3.5 \mathrm{mts}$. de alto.

- Casi todos tienen patios (o plazas).

- El tamaño de las estructuras aumenta, pero en comparación con las de Categoría 4 , son bastante pequeñas.

- En algunos existe pisos de estuco, tiestos policromados y piedra tallada. El residente se supone haber sido alguien con cierto poder político local. 


\section{Categoría 4}

Estos sitios tienen las características siguientes:

- La estructura más alta mide de 3.5 a 6 mts. de alto.

- Existe una plaza extensa delimitada por montículos pequeños a su alrededor.

- No tiene patrón de asentamiento tan complejo.

- En comparación con los de Categoría 3, las estructuras principales aumentan en tamaño notablemente.

\section{Categoría 5}

Son las unidades más grandes y se supone funcionaban como centros regionales. Sus características son:

- La estructura más alta alcanza de 5 a $12 \mathrm{mts}$. de alto.

- Varios grupos, de montículos, con su respectiva plaza de grandes dimensiones.

- Ocupa zona bastante extensa y tiene un patrón de asentamiento interno complejo.

- Aparecen elementos culturales mayas como estelas, jeroglíficos, construcción con bóveda, campo de pelota, etc.

\section{Categoría Especial}

Cementerios, estructuras sobre cerros, petroglifos, lugares de producción de cerámica y de lítica.

Conceptos Aplicados al Estudio Arqueológico Mediante nuevas Tecnologías de la Información Geográfica

\section{Sensores/Métodos Pasivos}

Instrumento que mide variaciones en la intensidad de radiación electromagnética producida por el Sol al interactuar con los objetos de la superficie terrestre. 


\section{Sensores / Métodos Activos}

Instrumento que mide variaciones en la intensidad de radiación electromagnética que él mismo emite en determinadas longitudes de onda y que puede registrar como respuesta al interactuar con los objetos de la superficie terrestre.

\section{GPR Ground Penetrating Radar.}

El geo-radar o radar de subsuelo, internacionalmente GPR (Ground Penetrating Radar), técnica de teledetección cercana (rango corto) y prospección basada en la emisión de un pulso electromagnético de muy corta duración (1-20 ns) caracterizado por una frecuencia nominal que puede oscilar entre los $10 \mathrm{MHz}$ y los 2,5 GHz, en función de la antena seleccionada. (Rejas, 2011).

Es una técnica no destructiva, no intrusiva, rápida y eficaz empleada en las investigaciones en profundidad de subsuelo, con buenos resultados para la detección de objetos enterrados. Prácticamente todo tipo de sepultura que cree un contraste de parámetros eléctricos es detectable con el GPR. Esto incluye parámetros de un cuerpo, parámetros de cualquier recinto de un cuerpo, y también el contraste en los parámetros del suelo creado por la excavación y relleno. Incluso una pequeña urna con las cenizas de la cremación crea un contraste detectable. GPR se ha demostrado satisfactoriamente para la localización de una gran variedad de lugares de enterramiento (Rejas, 2011).

\section{Estudio de Sitios Arqueológicos de Interés en Honduras.}

Se comenzó una investigación sistemática, en el Archivo Etnohistórico del $\mathrm{IHAH}$, sobre los diferentes sitios arqueológicos de Honduras. Se inició con una lista de sitios arqueológicos de El Paraíso, Valle de Sula, Islas de la Bahía, cuya información contenía coordenadas, municipio, mapas, etc. Se logró también información arqueológica de la Biosfera del Río Plátano (Olancho y Gracias a Dios). A pesar de los problemas que surgieron, en cuanto al acceso a la información, se logró información considerable.

Otra forma de lograr información sobre sitios arqueológicos de Honduras fue siguiendo como guía el artículo de Véliz (1983). Allí se divide la actividad arqueológica del país en cinco períodos, comenzando con los informes más tempranos y avanzando hacia los más recientes. Para cubrir información hasta el 2008, se agregó un sexto período. Los nombres y fechas que cubren los períodos son los siguientes: 1. Curiosidad 1576-1880, 2. Contacto 1880-1910, 3. Intimidad 19101950, 4. Receso 1950-1965, 5. Renacimiento 1965-1980 y 6. Esplendor 1980-2008. 


\section{Selección de Áreas Prioritarias de Estudio en Honduras}

En vista de la imposibilidad de poder cubrir todo el país desde el inicio, se decidió comenzar con ciertas zonas. No obstante, se hizo un esfuerzo inicial por recopilar la información sobre los sitios arqueológicos en el país; pero, debido a la poca información a nuestra disposición, fue poco lo que se logró para todo el país (Tabla 3).

El interés primordial era definir unas tres o cuatro zonas de interés para el IHAH y concentrar nuestros esfuerzos en esas zonas. Fue, en consulta con las autoridades del IHAH, cuando se logró definir tres zonas en el país. Además del interés del IHAH, para seleccionar estas zonas se tomó en cuenta el entorno natural, tratando de incluir zonas con entornos diferentes. El ensayo primordial en este proyecto es definir las características que en una imagen satelital representan un sitio arqueológico con estructuras visibles sobre la superficie terrestre. Ya que algunas zonas de estudio están bajo cultivo, se tendrá también la oportunidad de reconocer contrastes que aparezcan con respecto a diferencias de color del suelo. Las zonas seleccionadas fueron el Valle de Otoro, la Planicie Costera de Choluteca y una zona boscosa en Olancho, alrededor del Río Aner, afluente del Río Ahua Huas, el que finalmente desemboca en el Río Patuca (Figura 1).

\section{- Valle de Otoro}

Este valle está en el Departamento de Intibucá, Región Centro-Occidental del territorio hondureño, a pocos minutos hacia el oeste desde Siguatepeque, carretera a La Esperanza. Al Valle de Otoro lo baña y lo drena el Río del mismo nombre que, conocido como Río Puringla, nace en las Montañas de la Sierra, al sur, las que se extienden desde el Departamento de La Paz hacia el norte hasta el extremo sur del Departamento de Intibucá, donde está el valle. En Intibucá se le llama Río Grande o Río de Otoro y en el Departamento de Santa Bárbara se conoce como Río Jicatuyo, el que se convierte en el Río Ulúa, uno de los más importantes ríos de Honduras.

El Valle de Otoro tiene una extensión de $110 \mathrm{kms}^{2}$ y se aprovecha básicamente para la agricultura y la ganadería.

En este valle reina una vegetación de matorral, resultado de un bosque seco subtropical. El suelo del valle es arcillo-pedregoso y el tipo de plantas que alberga son resultado de una adaptación a la larga estación seca que reina en la 
zona. En este ambiente, muchas veces las hojas se transforman en espinas y, durante el verano, las hojas se caen. Los árboles son bajos y de madera dura.

La fauna que prevalece en este valle incluye: armadillos, conejos, zorrillos, comadrejas, lagartijas, garrobos, gatos de monte, ratones. Las aves que se encuentran en la zona son: pucuyo (pocuyo), alcaraván, tijul, paloma montera, chinchilote, lechuza de llano, codorniz (Pineda Portillo 1997: 170-171).

Arqueológicamente hablando, el valle es muy rico. De acuerdo con los estudios del IHAH (Cruz 2004), en $12 \mathrm{kms}^{2}$ se ha encontrado doce sitios arqueológicos, de los cuales diez son prehispánicos, uno es colonial y uno de petrograbados. De los prehispánicos, cinco son de Categoría 5 , lo que quiere decir básicamente que tienen estructuras de 5 a $12 \mathrm{mts}$. de altura y dos son de Categoría 4, con montículos de 3.5 a $6 \mathrm{mts}$. de altura. El resto son de tamaños menores (Ver Tablas 1, 2 y 3$)$.

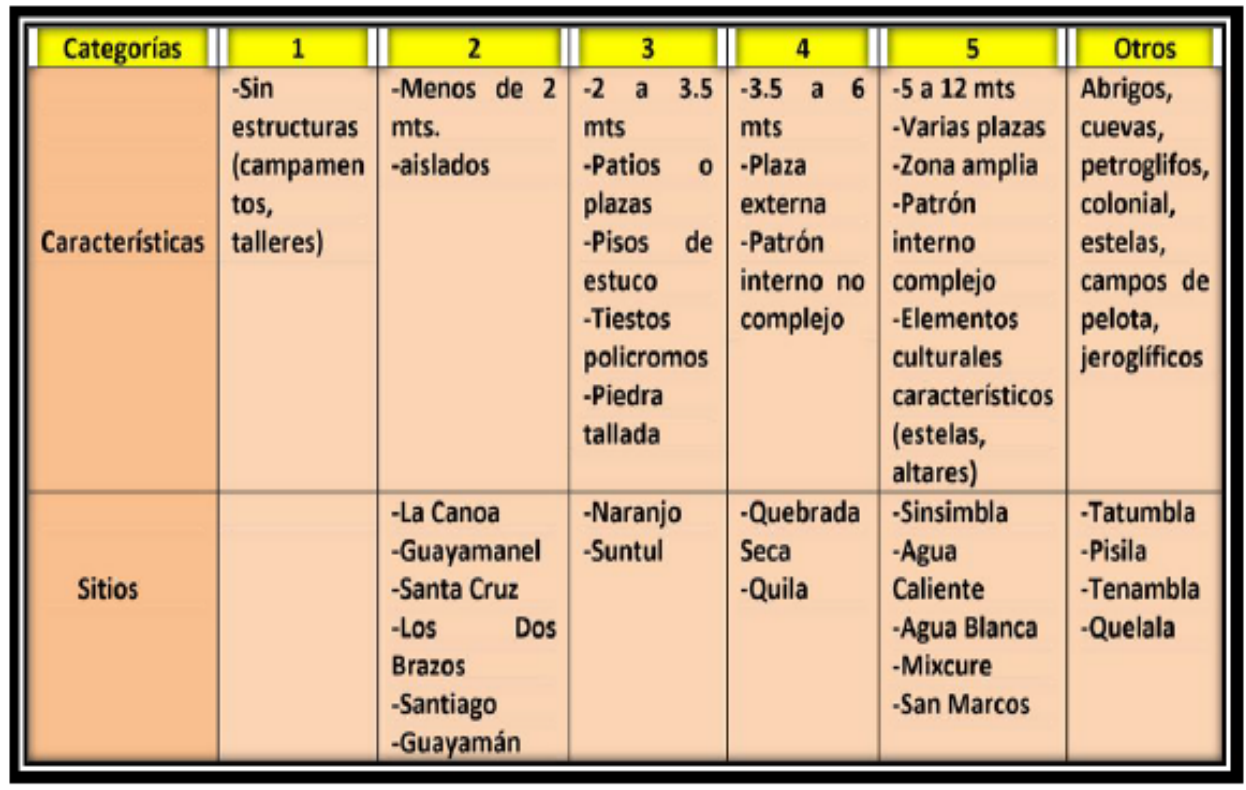

Tabla 1. Categorías arqueológicas de sitios en el Valle de Otoro. Según Vito, Veliz 


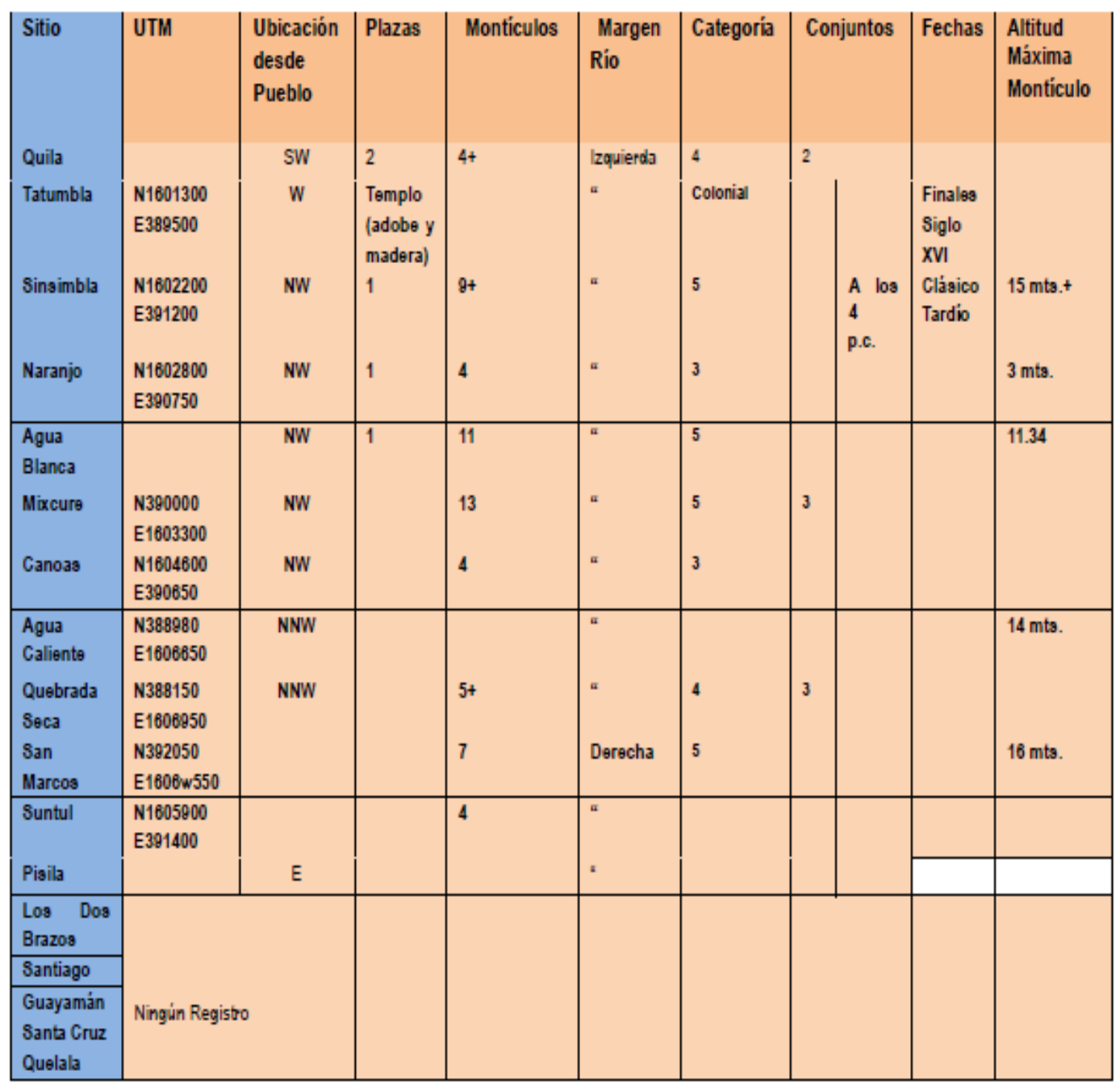

Tabla 2. Los Sitios Arqueológicos Registrados en el Valle de Otoro. Fuente IHAH 


\begin{tabular}{|c|c|c|c|c|c|c|c|c|c|c|c|c|c|c|c|c|}
\hline \multirow[b]{2}{*}{10} & & & & & & & & & & \multicolumn{2}{|c|}{ UTM } & \multirow[b]{2}{*}{ CIASIF WW } & \multirow[b]{2}{*}{ C.ARO } & \multirow[b]{2}{*}{ 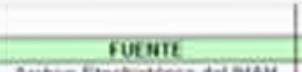 } & \multirow[b]{2}{*}{ AUTOR } & \multirow{3}{*}{$\begin{array}{l}\text { OASTRVACIOA } \\
\text { to Amedo Ramate? }\end{array}$} \\
\hline & SHAPE & HOsenf & OEMO & .......to & Mutacipto & $\cos m-1$ & PERLOOO & UAI & LONG & ESIE & HORIE & & & & & \\
\hline 1 & Punto & hodputes & hondationst & 11 & Rove in & 101 & E.tosos y & 160175 & अभत्र & 4409 & 1006 & Sotio & & 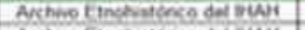 & fereminht fpeten & \\
\hline 3 & hanto & Consulathe & 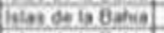 & "11 & Raven & 101 & Pondon & $16 \cdot 163 y$ & Coster $r$ & 5450 & 100150 & sane & & 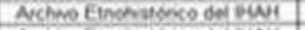 & Jereman f Epteren & 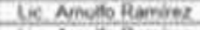 \\
\hline 3 & Pounco & Choredore & folen bo bollows & 11 & Rakan & 1107 & Bencoson $n$ & $16+160$ r & 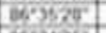 & $47 \times 0$ & 100000 & fatio & & 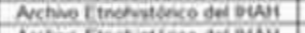 & Jetemon $r$ t potem & Le Anup Ramans. \\
\hline 4 & Panto & Aregues & tors of $10.8 \mathrm{ath}$. & in & Roven & 1101 & Peroes n & 162758 & $62435^{\circ}$ & 50000 & 101000 & selio & & 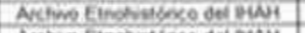 & Jerement Epstein & Le Anyph framez \\
\hline G & Preto & Dumporis & 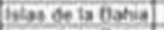 & 11 & Roxen & 1101 & Peroos n & 16025 & wron & 00000 & 10070 & the. & & 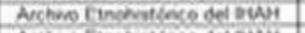 & Jetement r peten & IS Andero Ranter? \\
\hline s) & Anete & fowen Viata He & 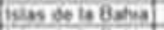 & II & Roum & 101 & Penosin & $1624 \times 2$ & $622113^{2}$ & 400 & 161030 & sene. & & 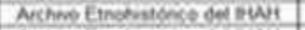 & Jeremanf Epten & US Amedo Revins \\
\hline & Deme. & 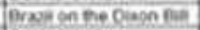 & thes oe in bithat & 11 & Bontin & 101 & Denos n & 167001 & $60121^{\circ}$ & $\$ 1000$ & 100590 & $\operatorname{sen} 6$ & & & dextench ipeten & Is Anedo Ravitite \\
\hline & Pato & Aspt the sato & fith on la 0 anias & 11 & Ravon & 1101 & henos $x$ & $1623 \times r$ & $16016 \times 0 \%$ & $\sin \infty$ & 181600 & $\sin 10$ & & 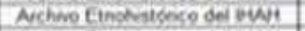 & Jeremonf t tpoten & We Amplo Ramerer \\
\hline & Deme & Comentey & then dolis Datis & il & Boxin & 101 & Bencon v & 16.1565 & 66014 & पीNos & 10240 & Sato. & & 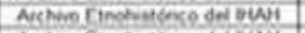 & Jeremon f Cettein & Wo Amodo Raminer \\
\hline & hento & Q2, & thindeta 8 otha & ii & Routon & 110 & Ponowe V & $1676 \times 1$ & 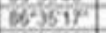 & 44000 & time & san & & 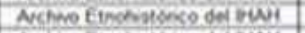 & Jeromon Epten & We Anots Ramins \\
\hline i1 & Pute & B. & 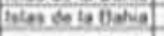 & 11 & Boxn & 1101 & Beredo in & 16232 & 62020 & 60200 & tones & Saio & & Asthe lombritore de our & devemph touten & Is Anud Ramier? \\
\hline 12. & Puote & BS & 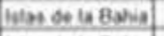 & 11 & Ravem & 1104 & Pented Vn & $16.154 \%$ & 050527 & 54700 & 100000 & Sate & & 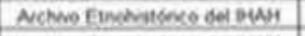 & sereminf F Esotein & Le. Almode Ramikes \\
\hline (1) & Puato & R.S & thas do 60 atises. & 11 & Rowain & 1100 & Pended on & 10240 & $6 x^{2} 4^{\circ}$ & 57600 & 161380 & Setio & & Acthoo Finchititoso del How & dermahf Epoten & Lic Anub Ramiser \\
\hline u. & Pare & R.19 & 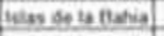 & ut & Ropis & 1101 & Perosen V & 16ras: & $04222^{\circ}$ & $\$ 9100$ & 100240 & sais & & 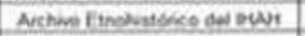 & 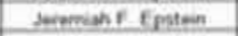 & is Aurb Rvonier \\
\hline 14. & Plento & B.H & 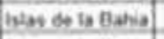 & "1 & Roven & 1101 & Peroes $\mathrm{n}$ & 152559 & $\operatorname{ces} 210$ & 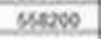 & 100330 & Ston & & 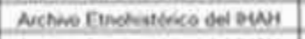 & deremonof Eproen & Le Amodo Ranister \\
\hline 16. $16 \mathrm{~T}-\mathrm{a}$ & Eunte. & $R+12$ & Wien ge ia Bana. & II & Roulan. & 1101 & Promon. & $16: 285 x$ & 062222 & 566900 & 161305 & 500. & & 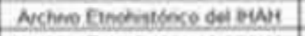 & Jertmohf Epoten & Ls. Anudo Ramiter. \\
\hline 11 & Aume & Q.4 & then do la 8 atiog & "I & Roxan & 1101 & Panosen V & $162+314$ & $362 \times 15$ & coszos & 10098 & Staio & & 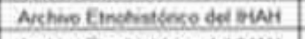 & Seremehf Eptoten & (N) Amudo Ramier \\
\hline (1) & Pate & R.15 & 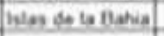 & 11 & Ravin & 101 & Putons VI & $1627 \mathrm{TC}^{-1}$ & $06360 t$ & $\operatorname{cosec}$ & 102970 & Satio & & Archmo findetinterse de buil & Jeremgan of tpatein & Lie Armato Ramiter \\
\hline 19 & Ponto & A.15 & when de be 8 ahis. & $\pi$ & Rouran & 1101 & Ponoto $\mathrm{C}$ & 162008 & $6525 x$ & $\$ 67200$ & to10e0 & ston & & 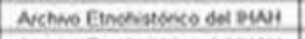 & deremin f Eptean & 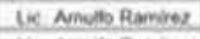 \\
\hline 20 & Punte & B.17 & 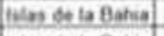 & 11 & Bowan & 1101 & Pencode $n$ & $16 \% 231^{2}$ & 25247 & 34090 & 161210 & So6. & & 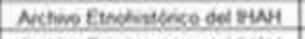 & Sermanf Epstein & Is Anuds Ramier. \\
\hline 21 & Douto & Q.10 & 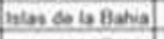 & "11 & Raxan & 1109 & Penodo VI & $1620 \times 1$ & 003121 & $\$ s 1000$ & 180590 & $\sin 0$ & & 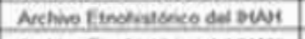 & Jermanf Epteen & Le Anuto Ramber \\
\hline$n$ & Punce & R.19 & 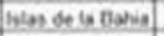 & 11 & Boxm & 101 & Penow $\mathrm{K}$. & 162125 & $00202 \pi$ & $\operatorname{sen} 00$ & 100000 & Savo & & 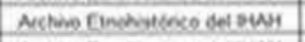 & Joremone fopten & Ge Amudo Ramiter \\
\hline$n$ & Aures & $18 \times 0$ & itures de in Benis. & 11 & Rowitan & $110 \%$ & Perode $\mathrm{W}$ & 162428 & $052320^{\circ}$ & sutos & 161980 & Satio. & & 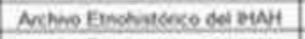 & Seremsanf Epeten & Us Anuito Ramiez \\
\hline 24 & Aunte & R21 & Wiens do to 8 shias. & II & Roven & 1101 & Penden $n$ & $162 y 3 r$ & $06212 x^{2}$ & 149700 & 101200 & Stio & & 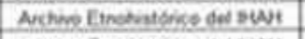 & deremiah f Epten & Is Amob Rumites \\
\hline 26 & Dento & $R \cdot n$ & hlas de la flatia. & II & Rowein & 1109 & Donosos $n$ & $162 \times 46$ & exirs & 576400 & 18160 & stion & & Nechen finchatereo del our & deremanf Coteen & Le Ansido Ramier \\
\hline$x$ & Anome & 2.28 & hitov de ia bana. & II & Rowhen & 1191 & Prodon & $1002 x+1$ & 062515 & $\$ 61909$ & 101300 & Soto & & 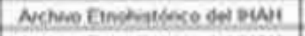 & Jeremine E Eptein & Is. Anowb Ramerez \\
\hline$z$ & Aumo & R.25 & Iniensola bakis & $" 1$ & Roxin & 1101 & Penden $\mathrm{V}$ & $167920^{\circ}$ & $66^{\circ} 1720^{\circ}$ & $\operatorname{sen} x$ & tores & Strig. & & 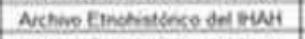 & Jereminf Epstan & LK Anups Ramits \\
\hline 20 & Parten & R.26 & Inese do bo Batia, & 11 & Ronan & 1101 & Punodo $\mathrm{V}$ & 16:avar & Qontro & 5,6500 & 184010 & Satio & & 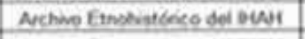 & Joremiahf Epotein & Le Amudo Ramier \\
\hline 20. & Dalo & 227 & helen de la forias. & 11 & Rowan. & 101 & Parnode $\mathrm{V}$ & $16.25+8$ & socires & 876200 & touto & Salis & & 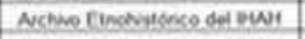 & decominf Epoten & the Anolb Romitez \\
\hline$x$ & Pento & R.28 & 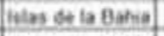 & 11 & Bowan & 1109 & Pended $\mathrm{W}$ & 152464 & 86.1650 & 576400 & 1840\% & Soro. & & 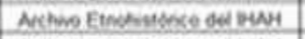 & Jeremanf Epstein & is Ampols Ramiez \\
\hline 31 & Purto & Rx & 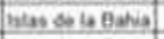 & "I & Roven & 1101 & Perado V & 16:3ar & Bot 642 & $57 \pi 000$ & 10466 & stio & & 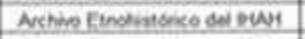 & Jeiemen F Eprean & Le Anuto Ramiez \\
\hline$x$ & Prento & a. 20 & Intar do to fakis. & $\pi$ & Rowan & 1101 & Derodos Vi & $1628 \mathrm{ar}$ & estrat & 675100 & 101480 & saip & & Acchion finchutione od bour & deteming fipten & tis Amado Ramiles \\
\hline 37 & Sores. & R.) & 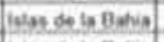 & 11 & Rowate & 1109 & Pende W & 161925 & 06305 & 47200 & 100470 & $\operatorname{san} 2$ & & 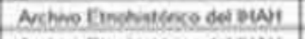 & dergman $\mathrm{r}$ Epeten & Us. Any/a Romity \\
\hline y & Punte & R.23. & lien as bosting. & II & Rowen & 101 & Penowin $\mathrm{V}$ & $16+185 y$ & oosys & $\$ 4400$ & 1802180 & Skin & & 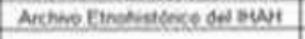 & Dereminf F Emten & Is Amus Renies \\
\hline & Banto & Dume Excosto & Cones: & os & Shen Pedros sus & esert. & Penowos n & & & napes & 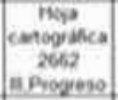 & tentition & & 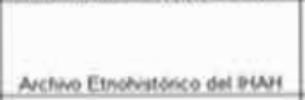 & 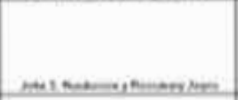 & (K. Anutb Raniez \\
\hline & some & Para de by Muntor & conts. & os & Ponierta & oset & Penoden & 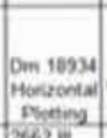 & 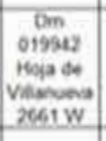 & new & & She & & 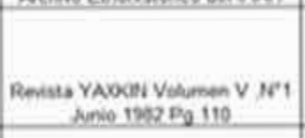 & Vhedenuac Kennety & Is. Amudo Bamiar \\
\hline & Perate. & castomes & conts. & es & Vormes & & Penots $\mathrm{V}$ & $\begin{array}{l}2002 \mathrm{~N} \\
2025 \mathrm{~N}\end{array}$ & 2325. w & & & sing & & 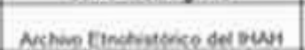 & $\sin A$ Baren & Iis Amolo Ramier \\
\hline 30 & heno & fornaryos & cones. & os & 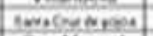 & $\operatorname{sic}$ & Porosen in & & & jonges & $16522+00$ & $\ldots+1$ & & 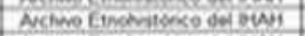 & & is Anoth Rarues \\
\hline $2 y$ & & tineoris & Conte & 8 & $\operatorname{sen}$ Noviel & $\infty 0$ & Pespoun & $2062=$ & 59291 & & & Stio & & 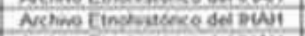 & 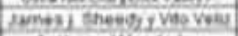 & is Anubs Romier \\
\hline 40 & Fure & tixes & conth & 05 & Smpotossta & Q⿻1 & Penosin & & & 21351608 & & Sitio & & Artho finchitosso del but & Antron Wonsider & Ls Anubs Ramites \\
\hline 41 & Punte. & cosin. & Cotes & os & Velanyera & osi1 & Penosin $V$ & $\left.{ }^{15}+1\right)^{\infty}$ & $2 s^{2} 00 \times 0$ : & & & Saig. & & Arthine Ethotriterso del BuH & Jum A Durth & Ls Amullo Raminet \\
\hline 4 & Ponto & $\cos p+(x)$ & $\operatorname{cosen}$ & 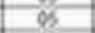 & cents & एक्ष & $\operatorname{pos} 0 \mathrm{x}$ & क्यका & $x \rightarrow x^{2}$ & & & Selin. & & 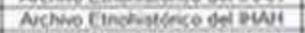 & 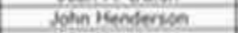 & 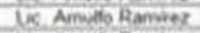 \\
\hline 4 & arese & If were & $\cos 103$ & $\infty$ & Snunued & oxs & Pungos V & $2662=1$ & 35004 & & & $\operatorname{sen} 0$ & & 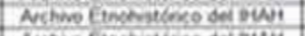 & Jenintersenven & Le Aneds Ramiers \\
\hline 4 & Arme. & prasesurek. & Coens & 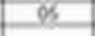 & frecontures & करी? & Pensole v & 26610 & 392610 & & & Stiog. & & 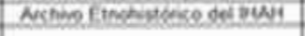 & $\sin A$ oron & Is Amios Ramies. \\
\hline is & Pente & reomeln & LPES & 12 & werar & 1261 & Periogs $n$ & 242648 & $\underset{n}{* 103 r o o r}$ & & & 50 & & 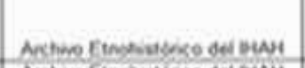 & & Lis Amato Ramier \\
\hline+6 & Counto & Cuma od Copute & GPN & 12 & Marais & 1200 & Peroson v & & & & & Sto & & Actives finchat tereo del but & 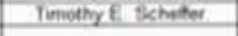 & Le Nuido Ramirer \\
\hline 47 & fruto. & Inpaza & Otanche & is & Cracamas & 1103 & Penosen $\mathrm{K}$ & $\frac{1469}{4 a^{2}}$ & $\left.058 t^{\prime} \leq 5\right]$ & & & insunte & & Nechere Einghatione del but & 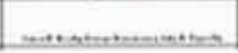 & Le Andots Ramatsz \\
\hline
\end{tabular}




\section{- Planicie Costera de Choluteca}

Esta zona está en la Región Sur de Honduras y la planicie costera del litoral Pacífico abarca un total del $2 \%$ del territorio nacional. Tiene una longitud de 133 kms. y la cruzan cuatro ríos, siendo estos, de oeste a este: Goascorán, Nacaome, Choluteca y Negro. El de mayor interés para nosotros en este momento y el de mayor caudal es el Río Choluteca. Formado por cuatro afluentes y dividiendo las ciudades gemelas de Tegucigalpa y Comayagüela, desde aquí se le conoce como Río Choluteca. Sigue rumbo norte, luego se dirige al este y por fin hacia el sur, teniendo una longitud de $250 \mathrm{kms}$ (Pineda Portillo 1997: 91).

Esta planicie costera goza de un clima de sabana, tropical lluvioso y seco, con altas temperaturas todo el año. De seis meses cada una, prevalecen dos estaciones bien marcadas: una de sequía (verano) de noviembre a abril y otra de lluvias (invierno) de mayo a octubre.

A partir del litoral y hasta los $600 \mathrm{msnm}$. Hacia el norte, la planicie goza de una vegetación de bosque seco tropical. El litoral es una unidad fisiográfica de limos y arcilla aluviales. La planicie está en la cadena de volcanes del Cuaternario, que viene desde Guatemala y se extiende hasta Panamá, pasando por el Golfo de Fonseca. Las islas (conos) de este golfo son testigo de esa actividad volcánica antigua.

En este litoral abundan los manglares, plantas que crecen a orillas del mar, en los estuarios formados por los ríos y las olas del mar. El mangle crece en suelo lodoso, proyectando una espesura de raíces aéreas. Al bajar las mareas, mucha vida acuática queda atrapada en esas raíces, aprovechándola otros animales terrestres y el humano aprovechando ambas, vida acuática y terrestre. Con sus raíces y los sedimentos atrapados, los manglares le van ganando terreno al mar y estos manglares son también una muy buena fuente de carbón vegetal.

Desde el punto de vista arqueológico, se tiene información general sobre salineras (Baudez s.f.) e información concreta de la existencia de dos sitios arqueológicos (Stone 1957: 97-98). Uno conocido como La Ola, un poco al noroeste de la Ciudad de Choluteca y se extiende a ambos lados del Río Choluteca; el otro sitio conocido como Colama, un poco al suroeste de la Ciudad de Choluteca. Ambos tienen montículos bajos de tierra y piedra. Se ha encontrado vasijas policromas. 


\section{- Zona Boscosa de Olancho, Río Aner}

Esta zona está en la Región Centro-Oriental del país, cerca de la unión de los Departamentos de: Olancho, Gracias a Dios y Colón. La vegetación y el clima son de bosque tropical lluvioso. La estación más lluviosa es entre los meses de septiembre a noviembre (Pineda Portillo 1997: 133-134). Ver Figura1.

Ésta es la zona menos conocida arqueológicamente y hasta el momento no ha sido posible ubicar unos pocos datos que manejan el Instituto Hondureño de Antropología e Historia.

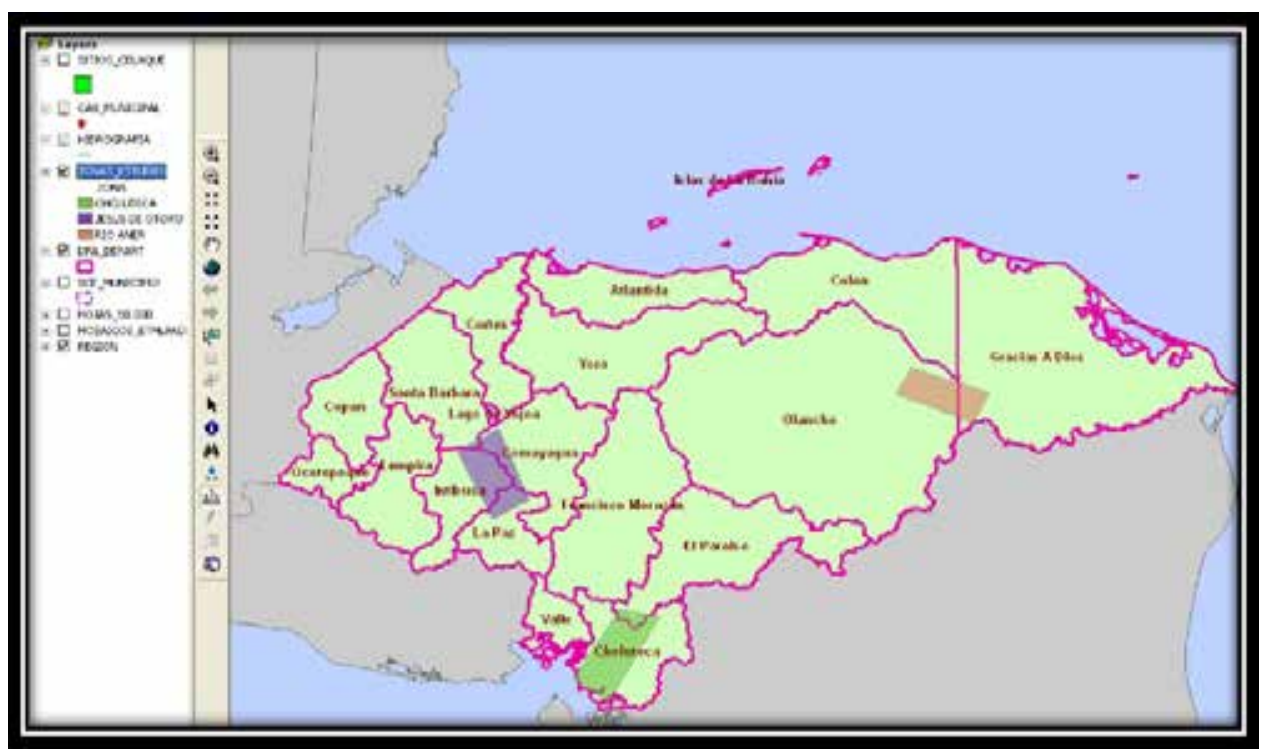

Figura 1. Zonas seleccionadas para la investigación. En color Verde Planicie Costera de Choluteca, en morado Valle de Otoro y en café Zona Boscosa de Olancho, Río Aner. Fuente CTIG-DAQAC.

El futuro de las investigaciones arqueológicas mediante Nuevas Tecnologías de la Información Geográfica en Honduras.

Una de las incontables tareas de la arqueología es el marcaje que se hace de los objetos que se obtienen en un sitio arqueológico. Se marca para poder manipular libremente los objetos. Siempre ha sido preocupante la cantidad de símbolos en los objetos arqueológicos, los que en muchos casos son pequeños y aparecen de mal gusto con tanta marca en su superficie. 
El IHAH ha comenzado a utilizar en sus registros arqueológicos, los mismos números que para los Departamentos y los Municipios ha venido utilizando el Registro Nacional de las Personas (RNP). De modo que, para los sitios del Valle de Otoro, estaremos utilizando el número 10, que es el que le corresponde al Departamento de Intibucá, según el orden alfabético de los 18 departamentos del país. Seguirá en este sentido luego el número 7 , el que alfabéticamente le corresponde al municipio de Jesús de Otoro. Por último se agregara el numero asignado para cada objeto (Montículo, altar, cerámica, lítica y hueso) estudiado en el sitio arqueológico. Lo anterior lo podemos resumir en la siguiente descripción arqueológica según: (\#departamento + \#municipio + \# de objeto encontrado). Por ejemplo (10 (Departamento de Intibucá)-7 (municipio de Jesús de Otoro) -120 (lítica)).

Otro ejemplo en Choluteca será primero el número 6, (según el orden alfabético de los 18 departamentos del país). Luego el número de municipio y al final el número asignado a cada objeto estudiado.

Vale la pena aclarar que, la numeración de los municipios de un departamento, comienza con aquel donde está la cabecera departamental y posteriormente se enumeran los municipios de cada Departamento en orden alfabético. Como otro ejemplo para la zona de estudio del Río Aner, se asignara el número 15, correspondiente al Departamento de Olancho, seguido por el número 5, representando al Municipio de Dulce Nombre de Culmí y, por último, el número de objeto en estudio.( (15 (Departamento de Olancho )-5 (municipio de Dulce Nombre de Culmí) -1550 (Cerámica utilitaria)).

La realidad es que, en la actualidad, se dispone de información concreta solamente para la zona del Valle de Otoro. En este valle, el IHAH ha venido realizando varias temporadas de campo y uno de los resultados más importantes para nuestros objetivos es la ubicación de sitios arqueológicos y los mapas de la mayoría de ellos. De uno de estos sitios, San Marcos, se obtuvo muestras de suelo para aplicar un análisis, conocer la reflectividad de este material y poder así ir comparando ya con lo que aparece en las imágenes satelitales. Ver figura 2.

Según los mapas disponibles, se puede ver ya alineamientos con los puntos cardinales, pues prácticamente todos los sitios y sus estructuras están dispuestos norte-sur. Esto nos lleva a concluir que puede haber alineamientos con los astros, en sus viajes de este a oeste.

Con estudiantes de la Asignatura Optativa "Introducción a la Arqueoastronomía", ya se hizo una primera visita a Jesús de Otoro para tratar de encontrar 
esas relaciones 0 alineamientos. Desafortunadamente, durante nuestra visita no fue posible corroborar nada, debido a la situación climática, pues tanto por la mañana como por la tarde no fue posible observar ni la salida ni la puesta del sol, astro con el cual deseamos iniciar nuestros estudios arqueoastronómicos.

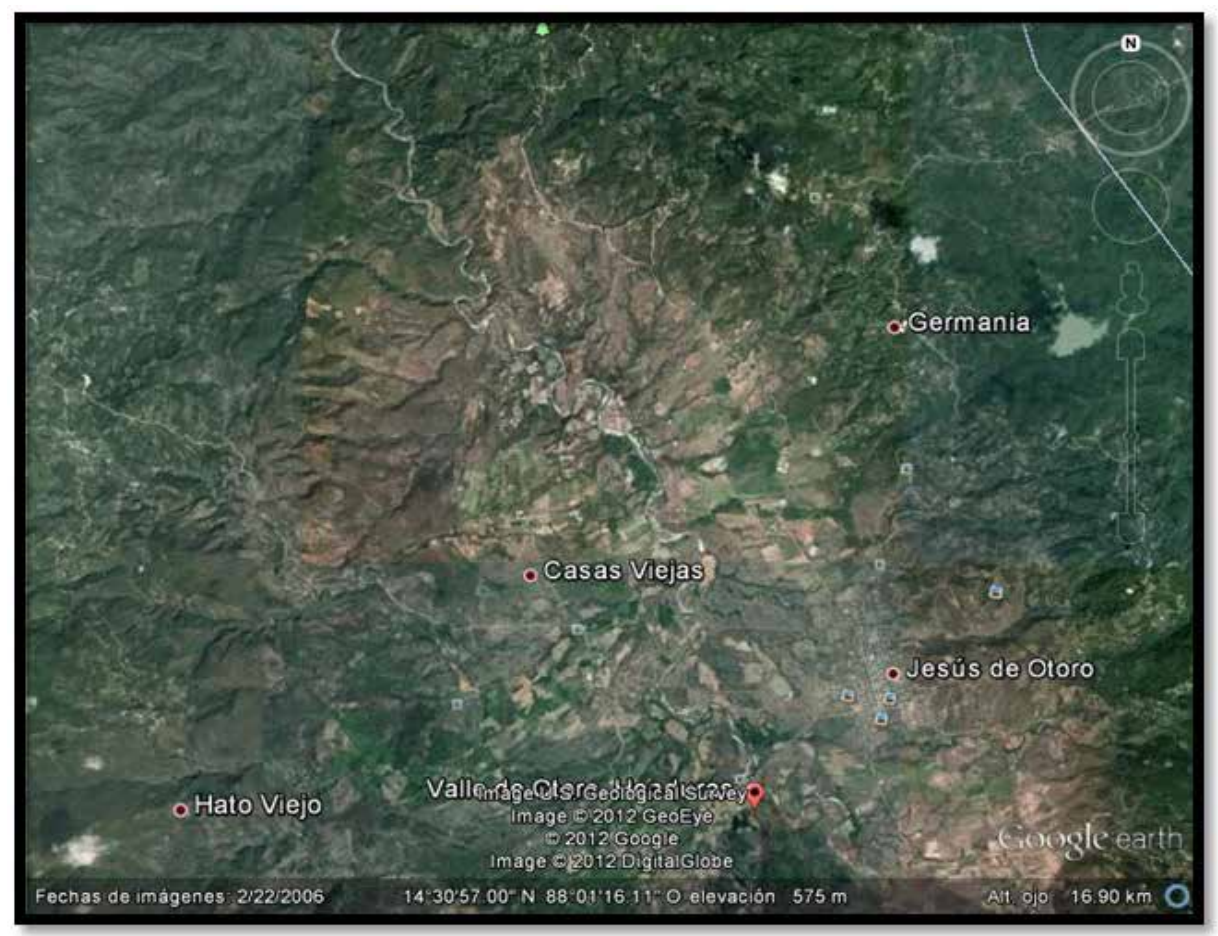

Figura 2. Muestra de imagen satelital de una de las zonas de estudio (Valle de Jesús de Otoro) obtenida de Google Earth para demostración.

\section{Conclusiones:}

Debido a la situación política en Honduras, el ritmo de este proyecto bajó. No obstante, tanto los investigadores españoles como los hondureños continuamos recopilando y organizando información. La búsqueda de información para la Identificación y localización de restos o sitios arqueológicos se intensificará y, en cuanto las condiciones climáticas y la disponibilidad de fondos lo permitan, estaremos saliendo al campo a recopilar la información pertinente sobre sitios arqueológicos visibles en la superficie y a tratar de reconocer esos sitios arqueológicos en las imágenes satelitales y a reconocer y a describir las características que esos sitios demuestran en las imágenes. 
Este proyecto ha permitido identificar técnicas modernas que ayudan a reconocer más rápidamente esos restos culturales en la superficie de la tierra. La técnica general de que se dispone es la teledetección, la que utiliza Sistemas de Información Geográfica, siendo la fuente de información los datos registrados por Sistemas de Observación de la Tierra, para estudio y para mejorar la protección del patrimonio cultural y de los hábitats humanos de Honduras ante, riesgos y desastres naturales y humanos.

Se realizó un Registro digital de los sitios arqueológicos. Pero además de ese registro, se espera estudiar el entorno para encontrar aquellas amenazas sobre los restos culturales. Esas amenazas pueden ser naturales (fuegos, inundaciones, huracanes) y/o antrópicas (proyectos de viviendas, agricultura, industria). Al identificar esos riesgos, se propondrá mecanismos de concientización y alternativas de uso de la tierra.

Se sabe que nuestros antepasados lograron mucho conocimiento astronómico y que mucho de ese conocimiento lo aplicaron en la distribución y orientación de monumentos dentro de los sitios arqueológicos y también entre sitios arqueológicos. La ubicación exacta de estos restos culturales en las imágenes satelitales servirá para encontrar esas relaciones en y entre sitios y la relación de monumentos y sitios arqueológicos con los astros.

\section{Bibliografía}

- Baudez, Claude F. s.f. Les Camps de Saliniers de la Cote Meridionale du Honduras: Donnees Archeologiques et Documents Historiques. En L'Homme, Hier et Aujourd'hui: Recueil d'Etudes en Hommage a Andre Leroi-Gourhan, (sin recopilador), pp. 507--519. Editions Cujas.

- Cruz, Oscar Neill. 2004. Patrón de Asentamiento Prehispánico en el Valle de Jesús de Otoro. VIII Seminario de Antropología Hondureña. Tegucigalpa.

- Estado de Honduras. 1997 Ley para la Protección del Patrimonio Cultural de la Nación (Decreto Legislativo 220-97). Instituto Hondureño de Antropología e Historia.

- Nakamura, Seiichi, Kazuo Aoyama y Eiji Uratsuji, Editores. 1991 Proyecto Arqueológico La Entrada, Primera Fase: Investigaciones Arqueológicas en la Región de La Entrada. Tomo I. Servicio de Voluntarios Japoneses para la Cooperación con el Extranjero e Instituto Hondureño de Antropología e Historia. Sociedad Nacional Papelera, San Pedro Sula. 
- Pineda Portillo, Noé.1997 Geografía de Honduras. Tercera Edición. Editorial Guaymuras, Tegucigalpa.

- Rejas, Juan Gregorio. 2011. Plataformas y Sensores en Prospección del Terreno. Tecnología GPR en excavación y recuperación de Restos Óseos. II Seminario Internacional de Antropología Forense, FOROST. Universidad Nacional Autónoma de México. Instituto de Investigaciones Antropológicas UNAM. México D.F., 28 de marzo de 2011. Consulta en: http://forost.org/seminar/Segundo_seminario/rejas_forost_2011.pdf

- Stone, Doris. 1957 The archaeology of central and southern Honduras. Papers of the Peabody Museum of Archaeology and Ethnology. Vol 49, No. 3. Cambridge, Massachusetts.

- Véliz, Vito. 1983 Síntesis histórica de la arqueología en Honduras. Yaxkin, Vol. VI, Nos. 1 y 2: 1-8. Órgano de Divulgación del Instituto Hondureño de Antropología e Historia, Tegucigalpa.

- Véliz, Vito, Rafael Corrales y Yeny Castellanos. 2009 Proyecto teledetección: Informe de Avance.

- UNESCO s.f. Documento de la convención sobre la protección del patrimonio mundial, cultural y natural.

- Willey, Gordon Randolph y Richard M. Leventhal. 1979 A preliminary report on prehistoric Maya settlements in the Copan Valley. En Maya Archaeology and Ethnology. Editores Norman Hammond y Gordon R. Willey, pp. 75-102. University of Texas Press, Austin. 